\title{
Noradrenaline Depletion Blocks Behavioral Sparing and Alters Cortical Morphogenesis after Neonatal Frontal Cortex Damage in Rats
}

\author{
Bryan Kolb and Robert J. Sutherland ${ }^{a}$ \\ Department of Psychology, University of Lethbridge, Lethbridge, Alberta, Canada T1K 3M4
}

\begin{abstract}
The possibility that cortical noradrenaline (NA) is necessary for sparing of function that occurs after neonatal frontal cortex damage was examined. Spatial localization by rats with frontal cortex damage on postnatal day 7 (P7) was better than that by rats with similar damage sustained as adults. The sparing was abclished in rats depleted of cortical NA by means of neonatal 6-hydroxydopamine (6HDA) administration. The blockade of sparing in the P7 frontal operates was associated with a smaller brain, thinner cortex, and reduced cortical dendritic branching relative to saline-treated P7 frontal operates. NA depletion alone in unoperated rats did not affect spatial learning but did reduce brain size and dendritic branching. Rats with frontal lesions on P4 did not show sparing of spatial localization, and 6HDA administration had no additional behavioral effect. Overall, these data are consistent with the notion that NA has some general function in maintaining some forms of plasticity in posterior cortex.
\end{abstract}

Frontal cortical damage sustained in rats at 7-10 days of age (P7-P10) allows significant sparing of behavioral function in adulthood relative to the effects of similar injuries sustained on P1-P5 or after P25 (e.g., Kolb, 1987). Although the basis of this functional sparing in P7-P10 animals is not known, several lines of work suggest that sparing after frontal cortical injury in rats is dependent upon neocortical circuitry in the remaining posterior cortex. For instance, Kolb and Whishaw (1981a,b) found that whereas frontal decortication at $P 7$ allowed significant sparing of function, complete decortication failed to allow functional sparing. Furthermore, Kolb and Gibb (1991a) found that behavioral sparing after frontal lesions at P10 is correlated with increased dendritic arborization throughout the layer II/III pyramidal cells of the posterior cortex. In contrast, frontal lesions at Pl were associated with even larger behavioral impairments than observed after similar lesions in adult rats, and this was correlated with a small decrease in dendritic arborization.

The possibility that cortical noradrenaline (NA) might pro-

\footnotetext{
Received Oct. 1, 1991; revised Jan. 8, 1992; accepted Jan. 15, 1992.

This research was supported by Natural Science and Engineering Research Council of Canada grants to each author and a National Centre for Exccllence grant to B.K. We acknowledge Grazyna Gorny and Robbin Gibb for their help with the Golgi drawings and Eddie Castenada and Robbin Gibb for doing the catecholamine assays.

Correspondence should be addressed to Bryan Kolb, Department of Psychology, University of Lethbridge, Lethbridge, Alberta, Canada T1K 3M4.

a Present address: Department of Psychology, University of New Mexico, Albuquerque, NM 87106.

Copyright (c) 1992 Society for Neuroscience $0270-6474 / 92 / 122321-10 \$ 05.00 / 0$
}

mote plasticity in the developing neocortex was first demonstrated by Kasamatsu and Pettigrew (1976). These authors found that depletion of NA from the kitten cortex by intraventricular 6-hydroxydopamine (6HDA) infusion blocked the shift in ocular dominance that is observed in visual cortical neurons after monocular deprivation during development (Kasamatsu and Pettigrew, 1976, 1979). It was later demonstrated that continuous local microperfusion of NA restores plasticity to the NAdepleted visual cortex (Kasamatsu et al., 1979). Furthermore, more recently it has been shown that direct infusion of $\beta$-adrenergic antagonists into the kitten visual cortex also suppresses the expected shift in ocular dominance after monocular lid suture (Shirokawa and Kasamatsu, 1986; Shirokawa et al., 1989). Although the findings of Kasamatsu and his colleagues have proven controversial (e.g., Gordon et al., 1988), they have stimulated research into the involvement of NA in other situations in which the cortex is modified either by experience (e.g., Pappas et al., 1978; Uylings et al., 1978; Brenner et al., 1983; O'Shea et al., 1983; Mohammed et al., 1986; Whishaw et al., 1986; Murtha et al., 1990) or by cortical injury in infancy (Sutherland et al., 1982; Castro et al., 1986; Whishaw et al., 1986; Kolb et al., 1989).

The object of the present series of experiments was to determine the relationship between neonatal NA depletion, behavioral sparing, and cortical morphology after frontal cortical lesions. Our initial report on this issue showed that perinatal NA depletion blocked behavioral sparing from frontal lesions at P7 but was without effect in adulthood (Sutherland et al., 1982). In view of our subsequent findings that behavioral sparing after $\mathrm{P} 10$ frontal lesions is correlated with increased cortical dendritic branching (Kolb and Gibb, 1991a), we wondered if the absence of cortical NA might prevent this plastic response and thus subsequently block sparing of function. We therefore replicated our original experiment and extended our observations by analyzing dendritic morphology in the same animals. In addition, since we knew that frontal lesions in the first few postnatal days do not allow behavioral sparing, we conducted an experiment to determine whether NA depletion affected the behavior and/ or cortical morphology of animals that would not be expected to exhibit sparing. Finally, since we had found that cortical thickness correlates with dendritic changes, we have availed ourselves of the opportunity to reanalyze the histological results of our original study, and these data are reported here as well.

\section{Materials and Methods}

Since the methods varied somewhat over the experiments, for clarity we will describe the methods of four experiments separately. However, since the results of the anatomical and behavioral measures in the dif- 
ferent experiments are complementary, we shall present the anatomical results of all four experiments together, followed by the behavioral results.

Experiment 1: Sutherland et al. (1982). Sixty Long-Evans hooded rats from seven litters bred at the University of Lethbridge served as subjects. Beginning within $24 \mathrm{hr}$ after birth, half of these rats received three daily injections of $6 \mathrm{HDA}(100 \mathrm{mg} / \mathrm{kg}$ in $0.9 \%$ saline and $0.2 \%$ ascorbic acid, s.c.) and half received only the vehicle solution. At 7-9 $\mathrm{d}$ of age, 30 rats were anesthetized using hypothermia and the medial frontal cortex was bilaterally aspirated according to previously described methods (Kolb and Whishaw, 1981b). Of these rats, 14 had received $6 \mathrm{HDA}$ and $16 \mathrm{had}$ received vehicle injections. The remaining rats were anesthetized and their scalps incised and sutured. Ten rats had the medial frontal cortex ablated at $100 \mathrm{~d}$ of age. Five of these animals had previously been given 6HDA treatments in infancy, while the others were given saline. Anesthesia was produced by $65 \mathrm{mg} / \mathrm{kg}$ of sodium pentobarbital in these adult animals.

Behavioral testing was conducted in the Morris water task (Morris, 1981) using procedures described in detail elsewhere (Sutherland et al., 1983). Briefly, the rats were trained to swim to a platform that was hidden just beneath the surface of $18^{\circ} \mathrm{C}$ water in a circular pool. The inside of the pool (diameter, $85 \mathrm{~cm}$; height, $45 \mathrm{~cm}$ ) was painted white, and the wire mesh surface of the platform was $1.5 \mathrm{~cm}$ below the water. Milk powder was dissolved in the water to render the platform invisible to a viewer at the water level. The rats began their search from one of four locations at the pool's perimeter, and the order of starting locations was randomly assigned. A group of four trials from each of the starting locations constituted a trial block, and there were two trial blocks per day. The platform remained in the center of one quadrant of the pool for 20 trials (five trial blocks) before being moved to the diagonally opposite quadrant for four trial blocks. We measured the latency to find the platform (maximum $120 \mathrm{sec}$ ), and one observer positioned beside the pool traced the swim path for each trial.

At the end of testing half of the rats were given an overdose of sodium pentobarbital, perfused intracardially with $0.9 \%$ saline followed by $10 \%$ formalin, processed for embedding in celloidin, and sectioned at $20 \mu \mathrm{m}$. Every 10 th section was mounted and stained with cresyl violet. Cortical thickness was measured by projecting the Nissl-stained sections on a Zeiss 2 POL projector set at a magnification of $13 \times$. According to the procedure described elsewhere (Kolb, 1987), measurements were taken at three different points at each of four planes corresponding to planes illustrated in Zilles (1985), Figures 13, 19, 22, and 26. The remaining half of the animals were killed for catecholamine assays as described in Sutherland et al. (1982).

Experiment 2 : postnatal day 7 replication. The animals were 29 LongEvans rats from three litters bred at the University of Lethbridge. The animals were treated with $6 \mathrm{HDA}$ or vehicle as in experiment 1 . Medial frontal $(n=13)$, medial frontal plus 6HDA $(n=8)$, or sham $(n=8)$ surgery was performed on the seventh postnatal day (P7) as described in experiment 1 .

Behavioral training in the Morris water task began at about $150 \mathrm{~d}$ of age in a larger tank (diameter, $1.5 \mathrm{~m}$ ) than in experiment 1 . The rats were trained for 13 trial blocks before the platform was moved to the diagonally opposite quadrant for one trial block. A video camera located above the pool tracked the trajectory of the rat, and the computer calculated the accuracy of the initial segment of the swim trajectory. Briefly, the computer drew an imaginary line from the starting location on each trial through the rat's position after it had traveled $16 \mathrm{~cm}$, and the deviation of this line from a secondary imaginary line from the starting location through the center of the platform was measured in degrees.

At the conclusion of behavioral testing the animals were weighed, given an overdose of sodium pentobarbital, and intracardially perfused with a solution of $0.9 \%$ saline followed by $10 \%$ formal saline. The brains were removed, weighed, and placed in 30\% sucrose formalin for at least $48 \mathrm{hr}$ before being cut frozen at $40 \mu \mathrm{m}$. Every 10 th section was saved and stained with cresyl violet. Cortical thickness was measured as in experiment 1 .

Experiment 3: postnatal day 4 lesions. The animals were 43 LongEvans rats from four litters from time-pregnant rats obtained from Quebec Breeding Farms. The animals were treated with $6 \mathrm{HDA}$ or vehicle as in experiment 1. Medial frontal $(n=6)$, medial frontal plus 6 HDA $(n=13)$, sham $(n=10)$, or sham plus 6 HDA $(n=14)$ surgery was performed on postnatal day 4 (P4) using the same methods as in experiment 1 .
Bchavioral training bcgan at about $150 \mathrm{~d}$ of age in the same maze as in experiment 2 . However, owing to the poorer performance of animals with frontal lesions before day 5 , the animals were trained for 24 trial blocks before the platform was moved for trial block 25 . As in the previous experiments, the latency to find the platform was recorded on every trial. In order to evaluate navigational accuracy, the swim path on each trial was drawn on a map of the pool and an error measure was later calculated: an $18 \mathrm{~cm}$ wide path from the start point to the platform was designated as the correct route and if a rat deviated from this route at any point, it received an error on that trial. At the completion of behavioral training the rats were killed and the brains processed as in experiment 2.

Experiment 4: postnatal day 7 Golgi. The animals were 45 LongEvans rats from five litters obtained from time-pregnant rats obtained from Qucbec Brecding Farms. The animals were treated with $6 \mathrm{HDA}$ or vehicle as in experiment 1 . Medial frontal $(n=15)$, medial frontal plus 6HDA $(n=18)$, sham $(n=6)$, or sham plus 6 HDA $(n=6)$ surgery was performed on postnatal day 7 (P7) using the same methods as in experiment 1 .

Behavioral testing was intended to be identical to experiment 2 , but owing to inadvertent experimenter error, the same procedure was not used for all of the groups, which invalidated the behavioral measure. These data are therefore not reported.

At the completion of behavioral testing (about $150 \mathrm{~d}$ of age), the animals were killed by decapitation. The brains were quickly removed and weighed, and then a small chunk $(2 \mathrm{~mm} \times 2 \mathrm{~mm})$ of the posterior part of Zilles Par 1 (Zilles, 1985) was removed from each hemisphere and used for an NA assay. The assay was performed using high-performance liquid chromatography with electrochemical detection (Felice et al., 1978; Robinson et al., 1987). The brain tissue was weighed and then homogenized in $400 \mu \mathrm{l}$ of $0.05 \mathrm{~N} \mathrm{HClO}_{4}$ containing $100 \mathrm{ng}$ of 3,4dihydroxybenzylamine hydrobromide as an internal standard and 0.8 $\mathrm{ml}$ of $\mathrm{M} \mathrm{NaHSO}_{4}$ as a reducing agent. A standard curve (tissue blank plus five standards containing known amounts of free-base NA) was run with each assay. Samples were centrifuged at $12,000 \times g$ for $5 \mathrm{~min}$. After centrifugation, all of the supernatant was transferred to conical tubes containing $20 \mathrm{mg}$ of acid-washed alumina and $200 \mu \mathrm{l}$ of $3 \mathrm{M}$ Tris$\mathrm{HCl}\left(\mathrm{pH} 8.6\right.$ at $4^{\circ} \mathrm{C}$ ). Samples were immediately vortexed for $1 \mathrm{~min}$ and shaken on a reciprocal shaker for $10 \mathrm{~min}$. Alumina was washed twice with $600 \mu \mathrm{l}$ of $6 \mathrm{mM}$ Tris-HCl $\left(\mathrm{pH} 8.6\right.$ at $\left.5^{\circ} \mathrm{C}\right)$ and twice with $600 \mu \mathrm{l}$ of $\mathrm{H}_{2} \mathrm{O}$. After completely removing the $\mathrm{H}_{2} \mathrm{O}$, the catecholamines were extracted with $200 \mu \mathrm{l}$ of $0.05 \mathrm{~N} \mathrm{HClO}_{4}$ by centrifugation for $2 \mathrm{~min}$. The supernatant was immediately assayed. The mobile phase was composed of 3 parts $0.1 \mathrm{~m}$ citric acid, 2 parts 0.1 м $\mathrm{NaHPO}_{4}, 0.0004 \%$ sodium octyl sulfate, and $15 \%$ methanol (pH 3.35). The mobile phase was pumped at $1 \mathrm{ml} / \mathrm{min}$ and continuously recycled. The detector potential was set at $0.72 \mathrm{~V}$ versus $\mathrm{Ag} / \mathrm{AgCl}$ reference electrode. Samples of $10 \mu \mathrm{l}$ were assayed with a sensitivity of $50 \mathrm{pg} / \mathrm{mg}$ for NA.

The rest of the brain was placed in a Golgi-Cox solution for $14 \mathrm{~d}$ before being sectioned at $200 \mu \mathrm{m}$ on a vibratome and then processed for Golgi-Cox staining using a procedure described by R. Gibb and $\mathbf{B}$. Kolb (unpublished observations). Ten layer II/III pyramidal neurons from Zilles area Par 1 and Te $2 / 3$ were drawn for each rat. In order to be included in the data analysis, the dendritic trees had to fulfill the following two criteria: (1) the cell had to be well impregnated and not obscured with precipitations, blood vessels, or heavy clusters of dendrites from other cells; (2) the cell had to lie approximately in the middle of the section thickness so that the apical and basilar dendrites were clearly visible in the plane of section. The cells were analyzed by drawing the cells via camera lucida, and then each branch segment was counted and summarized by branch order using the procedure of Coleman and Riesen (1968). Branch order was determined for the basilar dendrites such that branches originating at the cell body were first order; after one bifurcation, second order; and so on. Branch order was determined for the apical dendrites such that branches originating from the primary apical dendrite were first order, and so on. Cells were chosen by locating the areas in question and then by drawing each cell in that section that met the criterion listed above. Statistical analyses were performed by averaging across all cells per area per brain.

\section{Results}

Anatomical results

The seven main results were that (1) NA depletion decreased brain weight relative to vehicle treatment; (2) frontal lesions 
decreased brain weight at all ages, the effect being greater the earlier the lesion was made; (3) NA depletion and frontal lesions interacted to produce even smaller brains in male but not female rats; (4) neonatal frontal lesions reduced cortical thickness; (5) NA depletion in combination with adult or P7 lesions further reduced cortical thickness; (6) NA depletion decreased dendritic branching in both Par 1 and Te 2/3 in both control and frontal lesion groups; and (7) NA depletion and P7 lesions interacted to reduce dendritic branching even further. We shall consider brain weight, cortical thickness, and dendritic branching scparately.

General morphology. The frontal lesions included the medial prefrontal regions (Zilles $\mathrm{Cg} 1$ and $\mathrm{Cg} 3, \mathrm{FR} 2, \mathrm{PL}$, and IL) as well as variable amounts of motor cortex as illustrated in Figure 1. The adult and P7 lesions were comparable, although the P4 lesions were somewhat more extensive, which is a common finding (e.g., Kolb, 1987). Furthermore, as we have noted previously (e.g., Kolb, 1987) the thalamus was markedly smaller in the neonatal operates than in the control or adult frontal lesion groups. The lesions in the depleted and nondepleted rats appeared similar in extent, as did the thalamic shrinkage, although this impression was not quantified.

Brain weight. Table 1 summarizes the body and brain weight data, separated by sex, for experiments $2-4$. (These data were not available from experiment 1.) It can be seen that brain weight was reduced both by NA depletion and by P7 or P4 lesions in both sexes. Importantly, this reduction was even greater in every lesion +6HDA group. This latter effect appeared somewhat larger in males, however, a phenomenon that can be seen clearly in Figure 2, which summarizes the effects of brain weight data as a percentage of saline control animals. Since it seemed possible that this brain weight result might be related to body weight in some way, we did a similar analysis for body weight, which is summarized in Figure 3. The parallel with brain weight is striking, as NA-depleted males with frontal lesions are markedly smaller than similarly treated females. ANOVAs ( $p$ s $<0.05$ or better) confirmed both the brain weight and body weight results (see Table 1).

One puzzling inconsistency in the body weight results was that the P7 frontal lesions and P7 frontal lesions plus 6HDA had a much greater effect on body weight in experiment 4 than in experiment 2 . The only obvious differences between the experiments were that (1) the rats in experiment 4 were about 6 weeks younger at death than were those in experiment 2 , and (2) the rats in experiment 2 were born of females bred at the University of Lethbridge whereas the rats in experiment 4 were born from time-pregnant females obtained from Quebec Breeding Farms and shipped pregnant to the University of Lethbridge. Curiously, there was less discrepancy in brain weight than in body weight, and there was no discrepancy at all in cortical thickness (see below).

Cortical thickness. Neonatal frontal lesions previously have been shown to reduce the thickness of the remaining cortex (e.g., Kolb et al., 1983a), and this was again the case in all four experiments as summarized in Table 2. Thus, in order to facilitate cross-experiment comparisons, the data were converted to percentage of normal saline control group thickness, which is illustrated in Figure 4. A novel finding, however, was that NA depletion interacted with frontal lesions at $\mathrm{P} 7$ to reduce cortical thickness further in rats with P7 lesions. Somewhat surprisingly, a similar result was also observed in rats with frontal lesions in adulthood. In fact, the NA-depleted rats with adult
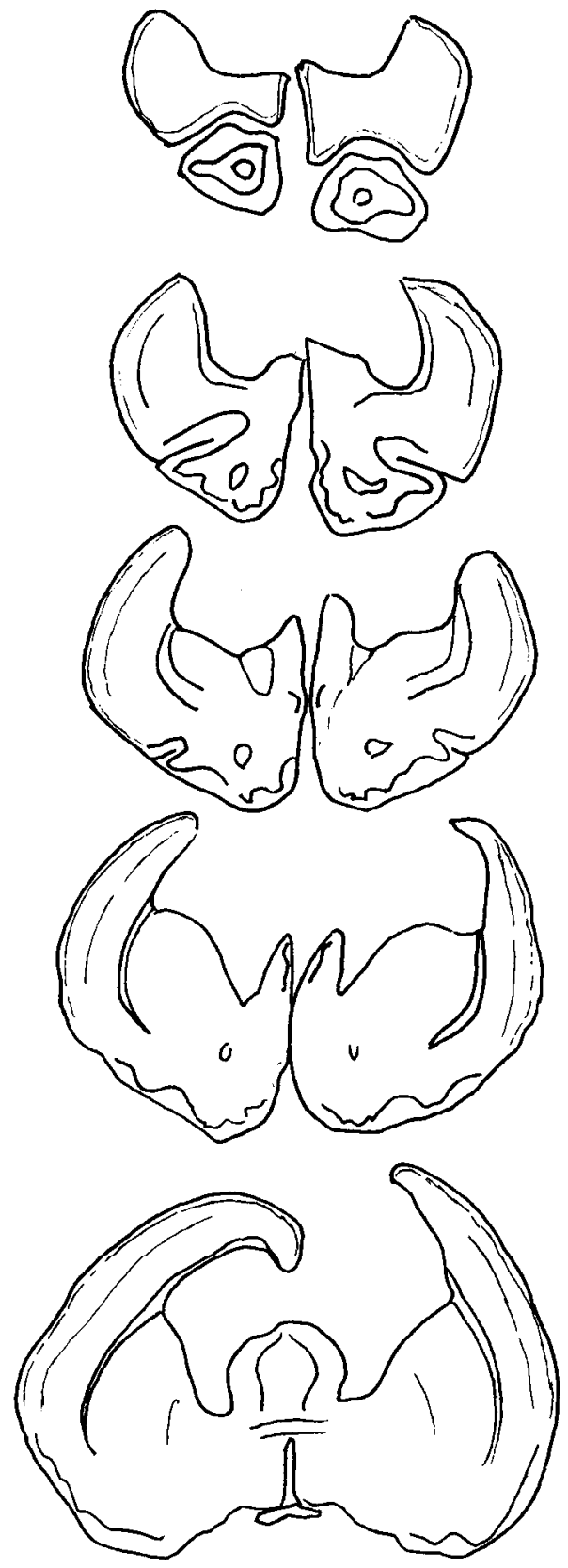

Figure 1. Serial constructions of a typical frontal lesion. Lesions usually included anterior cingulate areas, prelimbic and infralimbic regions, and supplementary motor cortex on the shoulder of the hemispherc.

frontal lesions had cortex that was as thin as animals with neonatal lesions. Unexpectedly, however, depleted rats with P4 lesions did not show a further decrease in cortical thickness, although the decrease in cortical thickness following a P4 lesion was substantially greater than after a P7 lesion, being in the order of $20 \%$.

There were no significant sex differences in any experiment, so the data for males and females were collapsed for ANOVA. Table 2 summarizes the ANOVAs for the main effects of group, all of which were significant. Follow-up tests (Fisher's LSD, $p$ s $<0.05$ ) showed significant neonatal lesion effects in all experiments and a significantly greater decrease in the P7 lesion +6 HDA groups in experiments 1 and 2 but not in experiment 4 (see Table 2). Although the reductions in cortical 
A Effect of noradrenaline depletion

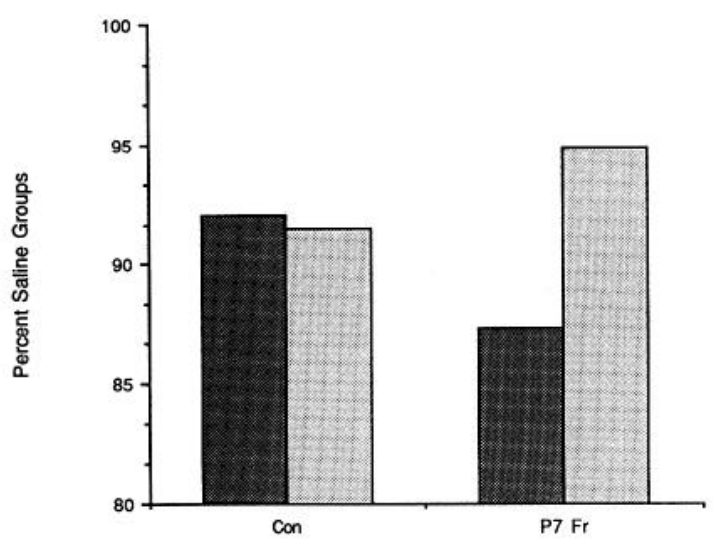

TREATMENT

B Effect of frontal lesion

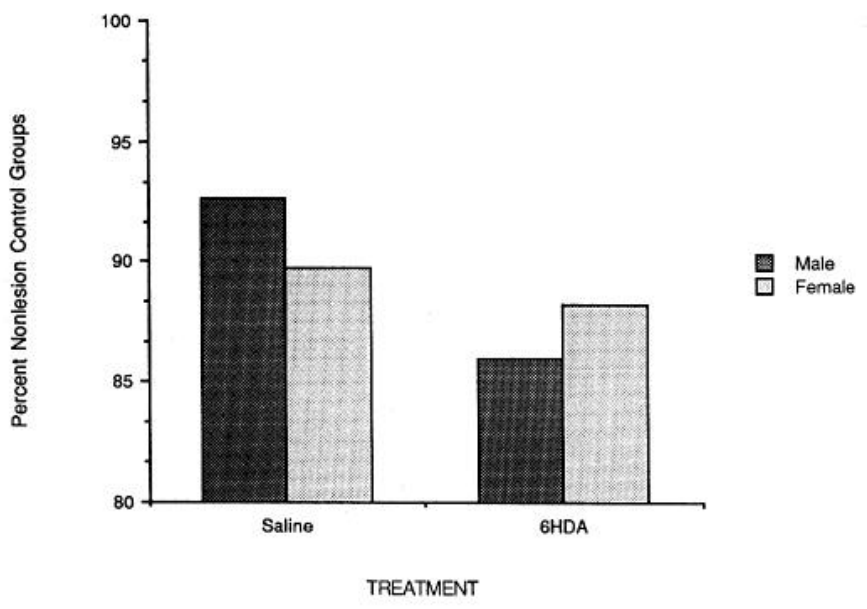

Figure 2. A, Summary of the effect of NA depletion on brain weight in experiments 2 and 4, expressed as a percentage of saline-treated control or frontal lesion groups, respectively. NA depletion reduced brain weight in both operated and unoperated rats and had the greatest effect in male frontal operates. Con, unoperated control rats; $P 7 \mathrm{Fr}$, rats with frontal lesions on P7. B, Summary of the effect of P7 frontal lesions on brain weight in experiments 2 and 4, expressed as percentage of unoperated control groups. Saline-treated frontal operates had smaller brains than controls, and 6HDA-treated male but not female frontal rats had a further reduction in brain weight. $6 \mathrm{HDA}, 6 \mathrm{HDA}$-treated rats with frontal lesions on P7; Saline, saline-treated rats with frontal lesions on P7.

thickness generally appeared to be greater in the more rostral sections, follow-up ANOVAs at each plane were significant in each experiment ( $p s<0.05$ ), with the exception of plane 3 in experiment 2 .

Dendritic branching. The general finding was that NA depletion reduced dendritic branching in both the apical and basilar dendrites in both parietal and temporal cortex (Figs. 5-7), and that the effect was increased in rats with $\mathrm{P} 7$ frontal lesions. The neurons in the depleted rats appeared generally normal in structure, however, with no evidence of stunted or malformed neurons. Separate ANOVAs were done on the apical and basilar dendrites. For the parietal cortex, there were significant effects of group $(F=7.7, p<0.01 ; F=8.3, p<0.01)$ and NA depletion $(F=37.7, p<0.001 ; F=53.9, p<0.001)$ for both the apical and basilar branches, respectively. The interaction only reached
Table 1. Summary of body and brain weights in experiments 2-4

\begin{tabular}{|c|c|c|c|c|}
\hline \multirow[b]{2}{*}{ Group } & \multicolumn{2}{|c|}{ Body weight (\%) } & \multicolumn{2}{|c|}{ Brain weight $(\%)$} \\
\hline & Male & Female & Male & Female \\
\hline \multicolumn{5}{|l|}{ Experiment 2} \\
\hline Control & 100 & 100 & 100 & 100 \\
\hline P7 frontal & 98 & 98 & $95^{*}$ & $92^{*}$ \\
\hline P7 frontal+6HDA & 90 & 95 & $85^{*}$ & $88^{*}$ \\
\hline \multicolumn{5}{|l|}{ Experiment 3} \\
\hline Control & 100 & 100 & 100 & 100 \\
\hline Control+6HDA & $89^{*}$ & $75^{*}$ & $93^{*}$ & $91^{*}$ \\
\hline P4 frontal & 101 & 95 & $79 *$ & $83^{*}$ \\
\hline $\mathrm{P} 4$ frontal $+6 \mathrm{HDA}$ & $71^{*}$ & $81^{*}$ & $69^{*}$ & $77^{*}$ \\
\hline \multicolumn{5}{|l|}{ Experiment 4} \\
\hline Control & 100 & 100 & 100 & 100 \\
\hline Control+6HDA & $89^{*}$ & $88^{*}$ & $91^{*}$ & $92^{*}$ \\
\hline P7 frontal & $90^{*}$ & $81^{*}$ & $90^{*}$ & $86^{*}$ \\
\hline P7 frontal+6HDA & $68^{*}$ & $71^{*}$ & $77^{*}$ & $78^{*}$ \\
\hline
\end{tabular}

Numbers are expressed as percentage of the saline-treated control group in each experiment. P4 frontal, rats with frontal lesions on P4; P7 frontal, rats with frontal lesions on P7. 6HDA indicates groups that received 6HDA lesions.

* Significant difference from the saline-treated control (Fisher's LSD, $p \mathrm{~s}<0.05$ or better).

significance for the basilar branches, however $(F=1.3, p=0.25$; $F=4.8, p=0.03$, respectively). Follow-up tests showed that nondepleted control and $\mathrm{P} 7$ lesion groups did not differ in either apical or basilar branches whereas these comparisons were significant for the NA-depleted groups.

ANOVAs for the temporal cortical branching showed there were significant group $(F=4.7, p=0.03 ; F=6.0, p<0.02)$ and NA depletion $(F=15.6, p<0.001 ; F=8.3, p<0.01)$ effects for the apical and basilar branches, respectively. The interactions were not significant in either case $(p s>0.4)$. Followup tests again failed to show a lesion effect, but the P7 NAdepleted rats had fewer basilar branches than NA-depleted controls.

\section{Neurochemical results}

Bioassays were performed on animals from experiments 1 and 4, with the two principal results being that (1) rats treated with 6 HDA had over $96 \%$ depletions of cortical NA and (2) frontal lesions alone produced about $50 \%$ depletions in cortical NA (Table 3). ANOVAs showed group effects in both experiment 1 and experiment $4(F=48.8, p<0.001 ; F=25.5, p<0.001)$. Follow-up comparisons (Fisher's LSD, $p \mathrm{~s}<0.01$ ) showed significant differences between the depleted and nondepleted animals in both control and operated groups as well as significant differences between nondepleted control and frontal-operated animals.

\section{Behavioral results}

The four principal behavioral results were that (1) rats with medial frontal lesions at P7, but not at P4, showed significant sparing of spatial learning relative to similar lesions in adulthood; (2) cortical NA depletion eliminated the sparing in rats with P7 lesions; (3) cortical NA depletion was without noticeable effect on rats with adult or P4 lesions, and (4) unoperated rats with cortical NA depletion did not differ in any respect from normal control animals.

The behavior of the control animals in the spatial localization 
A Effect of noradrenaline depletion

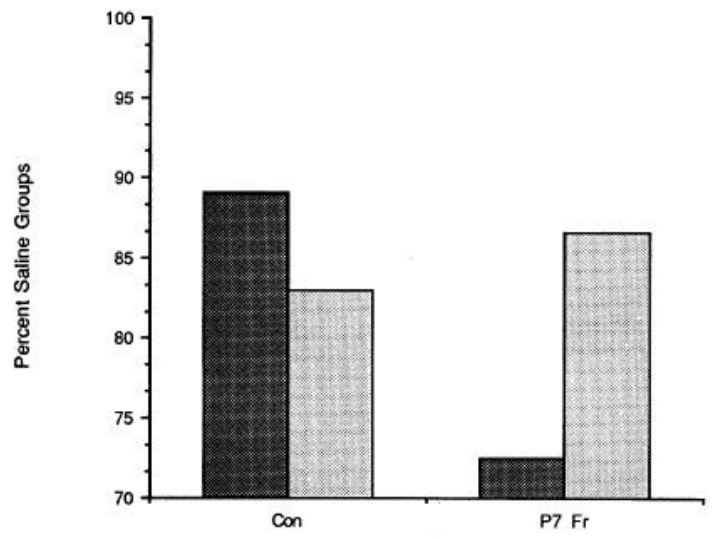

TREATMENT

B Effect of frontal lesion

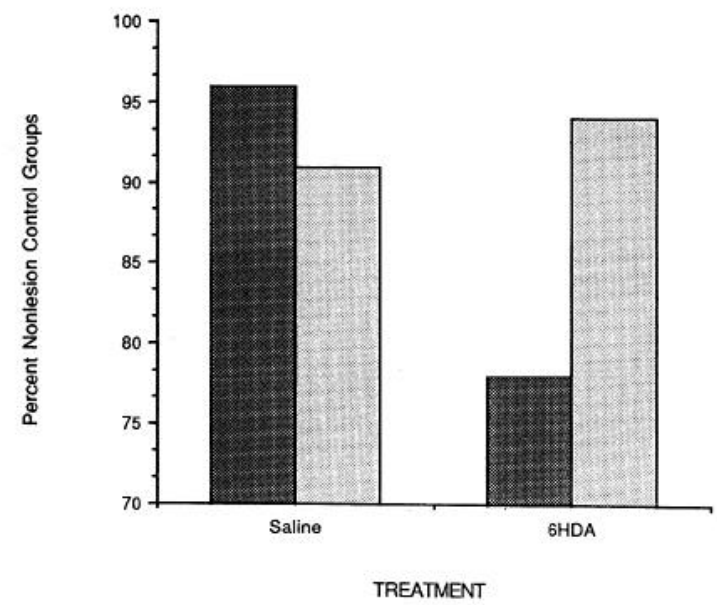

Figure 3. A, Summary of the effect of NA depletion on body weight in experiments 2 and 4 , expressed as a percentage of saline-treated control or frontal lesion groups, respectively. NA depletion reduced body weight in both operated and unoperated rats but had the biggest effect on male frontal operates. Con, unoperated control rats; $P 7 \mathrm{Fr}$, rats with frontal lesions on P7. B, Summary of the effect of P7 frontal lesions on body weight in experiments 2 and 4 , expressed as percentage of unoperated control groups. Saline-treated frontal operates were lighter than controls, and 6HDA-treated males, but not females, showed the greatest relative reduction in body weight. $6 H D A, 6 \mathrm{HDA}$-treated rats with frontal lesions on $\mathrm{P} 7$; Saline, saline-treated rats with frontal lesions on P7.

task in each experiment was very similar to previous reports (e.g., Sutherland et al., 1983). After the first block of trials, they reliably swam directly to the platform, and when the platform was moved they took longer to find it since they persisted in swimming in the previously correct quadrant of the pool (Fig. 8). Rats with medial frontal lesions in adulthood or at P4 swam normally in the pool but were slower to find the platform initially and were unable to learn to swim directly to it, although their escape latency did improve with training. Rats with P7 lesions showed an initial impairment in finding the platform but performed as well as control animals within five trial blocks. Neonatal NA depletion completely blocked this sparing, however, in both experiments 1 and 2, as the depleted animals were unable to learn the location of the platform, although they swam nor-
Table 2. Summary of cortical thickness measurements

\begin{tabular}{llc} 
Group & $\begin{array}{l}\text { Cortical thickness } \\
(\mathrm{mm})\end{array}$ & $\begin{array}{l}\text { Percent- } \\
\text { age of } \\
\text { control }\end{array}$ \\
\hline Experiment 1 & $1.08 \pm 0.005$ & 100 \\
$\quad$ Control & $1.08 \pm 0.006$ & 100 \\
Control+6HDA & $1.08 \pm 0.012$ & 100 \\
Adult frontal & $0.99 \pm 0.018$ & 92 \\
$\quad$ Adult frontal+6HDA & $1.02 \pm 0.012$ & 94 \\
P7frontal & $0.97 \pm 0.023$ & 90 \\
P7 frontal+6HDA & $1.35 \pm 0.029$ & 100 \\
Experiment 2 & $1.28 \pm 0.029$ & 95 \\
Control & $1.17 \pm 0.024$ & 87 \\
P7 frontal & $1.76 \pm 0.021$ & 100 \\
P7 frontal+6HDA & $1.81 \pm 0.050$ & 103 \\
Experiment 3 & $1.35 \pm 0.035$ & 77 \\
Control & $1.35 \pm 0.074$ & 77 \\
Control+6HDA & & \\
P4 frontal & $1.95 \pm 0.050$ & 100 \\
P4 frontal+6HDA & $1.94 \pm 0.024$ & 100 \\
Experiment 4 & $1.84 \pm 0.017$ & 94 \\
Control & $1.78 \pm 0.012$ & 91 \\
Control+6HDA & & \\
P7 frontal & & \\
P7 frontal+6HDA & &
\end{tabular}

Thickness numbers represent means and SE. Percentage control values are percentage of saline-treated controls in each experiment. P4 and P7 frontal refers to groups with frontal lesions on $\mathrm{P} 4$ or $\mathrm{P} 7$, respectively. $6 \mathrm{HDA}$ indicates groups that received $6 \mathrm{HDA}$ lesions.

mally and actively searched for the platform (Fig. 8). That is, the depleted animals learned that there was an escape platform and learned a strategy to find it, but they did not learn its spatial location and in that sense behaved like decorticated rats (Kolb et al., 1983b). NA depletion did not affect the performance of rats with $\mathrm{P} 4$ lesions or unoperated rats (Fig. 9).

Statistical analyses on the latency scores confirmed these observations, as overall ANOVA revealed significant group effects (experiment 1: $F=4.8, p<0.002$; experiment 2: $F=3.34, p$ $=0.05$; experiment $3: F=13.9, p<0.001$ ), trial effects (experiment $1: F=23.7, p<0.001$; experiment $2: F=42.7, p<$ 0.001 ; experiment $3: F=31.1, p<0.001)$ and interaction effects (experiment 1: $F=2.6, p<0.03$; experiment $2: F=3.1, p<$ 0.001 ; experiment $3: F=5.8, p<0.001$ ), respectively. Followup comparisons (Fisher's LSD, $p s=0.05$ or better) showed that

Table 3. Summary of NA assays of posterior cortex in experiment 4

\begin{tabular}{llc} 
Group & Cortical NA (ng/mg) & $\begin{array}{l}\text { Percent- } \\
\text { age of } \\
\text { control }\end{array}$ \\
\hline Control & $0.263 \pm 0.067$ & 100.0 \\
Control+6HDA & $0.006 \pm 0.002^{*}$ & 2.3 \\
P7 frontal & $0.152 \pm 0.010^{*} \dagger$ & 57.8 \\
P7 frontal+6HDA & $0.005 \pm 0.001^{*}$ & 1.9
\end{tabular}

Cortical NA numbers represent means and SE. Percentage control refers to percentage of saline-treated control group. P7 frontal refers to rats with frontal lesions on P7. 6HDA indicates groups that received 6HDA lesions.

* Differs from control group $(p<0.01)$.

$\uparrow$ Differs from all groups $(p<0.01)$. 
A Adult and P7 Lesions

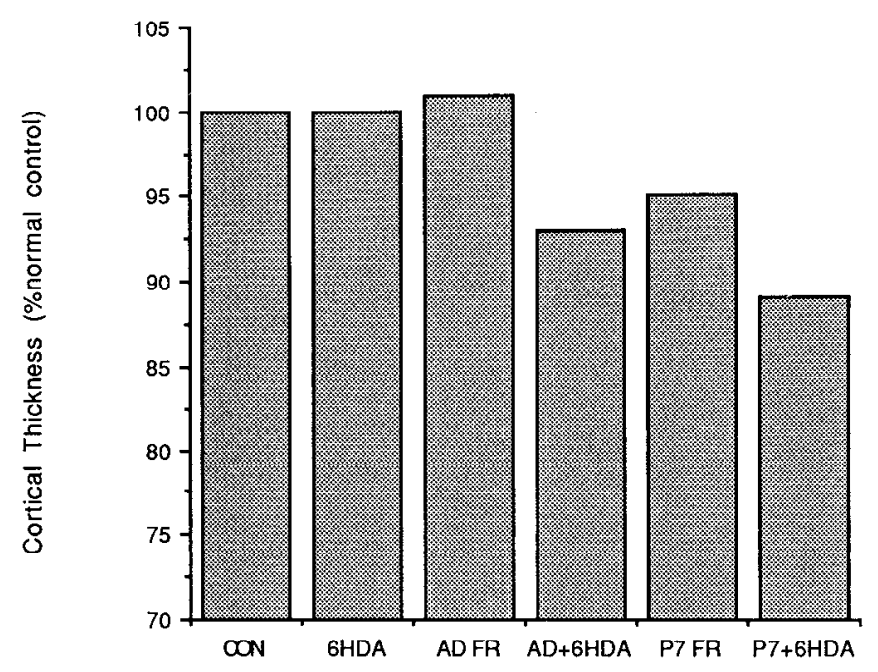

GROP

\section{B P4 Lesions}

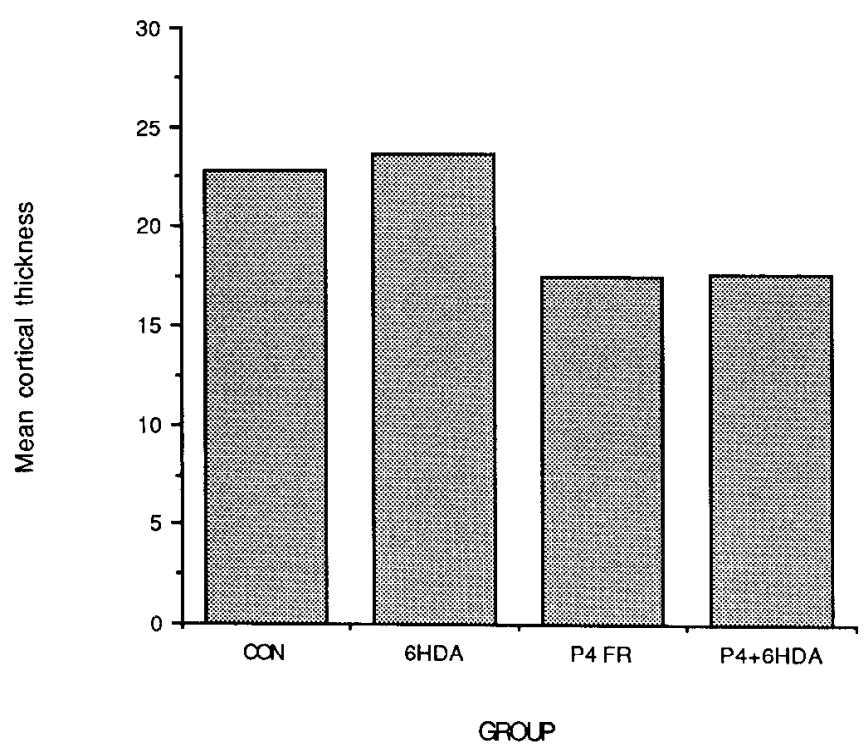

Figure 4. A, Summary of cortical thickness measurements in experiments 1,2 , and 4 expressed as a percentage of normal control thickness. Rats with frontal lesions in adulthood showed no change in cortical thickness unless they were given 6HDA treatment in infancy. P7 frontal lesions $(P 7 F R)$ reduced cortical thickness, and the addition of 6HDA treatment further decreased cortical thickness. $B$, Summary of cortical thickness measurements in experiment 3 . Frontal lesions reduced cortical thickness but 6HDA had no additional effect. $C O N$, saline-treated unoperated control rats; $6 H D A, 6 \mathrm{HDA}$-treated unoperated control rats; $A D F R$, adult-operated frontal lesion rats; $A D+6 H D A$, adult-operated frontal lesion rats who received $6 \mathrm{HDA}$ in infancy; $P 7 F K, \mathrm{P} 7$ frontal lesion rats; $P 7+6 H D A, \mathrm{P} 7$ frontal lesion rats treated with $6 \mathrm{HDA} ; P 4$ $F R, \mathrm{P} 4$ frontal lesion rats; $P 4+6 H D A, \mathrm{P} 4$ frontal lesion rats treated with $6 \mathrm{HDA}$.

(1) all lesion groups differed from control groups, (2) the P7 lesion and $\mathrm{P} 7$ lesion $+6 \mathrm{HDA}$ groups differed from one another,

(3) the P4 lesion and P4 lesion +6HDA groups did not differ,

(4) the adult frontal lesion and adult lesion +6 HDA groups did not differ, and (5) the control and 6HDA groups did not differ.

\section{A NORMAL}
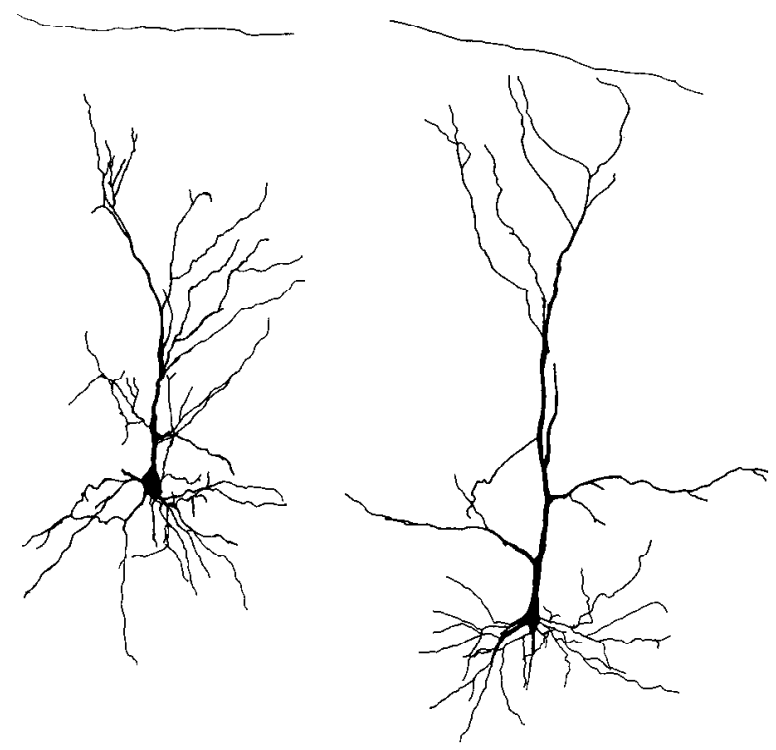

\section{B 6-HDA}
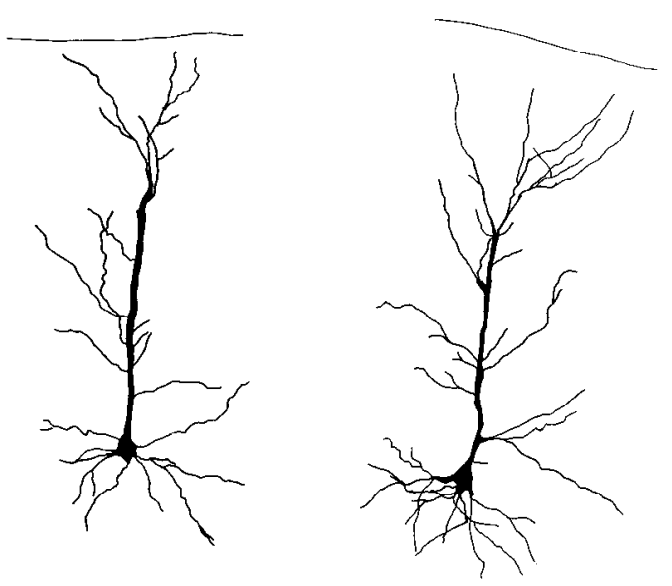

Figure 5. Drawings of representative layer II/III pyramidal cells from Par 1 in a saline-treated unoperated rat $(N O R M A L)$ and a rat with $6 \mathrm{HDA}$ treatment and a frontal lesion on $\mathrm{P} 7(6-H D A)$. The $6-H D A$ cells are less branchy than the control cells but do not appear to be stunted or grossly misshapen.

ANOVA on the heading angles on trial blocks $1,5,9$, and 13 in experiment 2 found a significant group effect $(F=5.9, p=$ $0.008)$ and practice effect $(F=35.0, p<0.001)$ but no interaction $(F=1.2, p=0.29)$. Follow-up comparisons showed that, whereas there were no differences in heading orientation on the first trial block, the control and P7 lesion +6 HDA groups differed significantly on the trial block $13(p<0.01)$, whereas the P7 lesion +6HDA group failed to differ from any other group.

ANOVA on the heading errors in experiment 3 showed a significant group effect $(F=38.7, p<0.001)$, practicc effect $(F$ $=25.3, p<0.001)$, and interaction $(F=3.1, p<0.001)$. Post hoc tests ( $p \mathrm{~s}=0.05$ or better) revealed that the control and $6 \mathrm{HDA}$ groups differed from the two lesion groups, which did not differ from one another. 
AREA PAR 1 APICAL DENDRITES

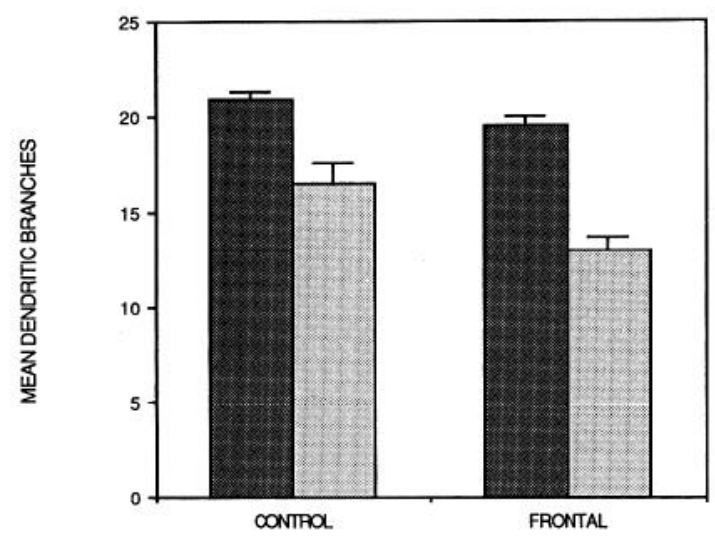

GROP

AREA PAR 1 BASILAR DENDRITES

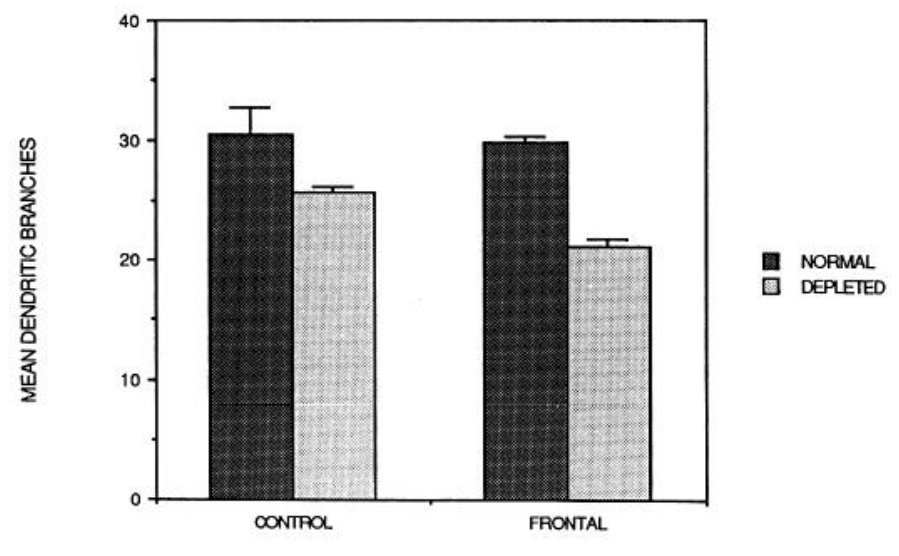

GROP

Figure 6. Mean number of apical and basilar dendritic branches for pyramidal cells in layer II/III of Par 1 . The 6HDA treatment $(D E$ PLETED) reduced branching in both control and neonatal frontal lesion groups, but the effect was greater in the frontal lesion group.

\section{Discussion}

These results clearly confirm that neonatal cortical NA depletion blocks the behavioral sparing normally observed in rats with frontal lesions at P7. The principal novel findings were that this blockade of sparing in the NA-depleted frontal operates was associated with a smaller brain and thinner cortex, as well as a greater reduction in dendritic branching than was observed in saline-treated frontal operates. Further, the present experiments showed that NA depletion did not affect the behavior of normal animals, animals with frontal lesions at P4 or animals with frontal lesions in adulthood. Hence, NA depletion only affected the spatial learning performance of rats that would normally have shown significant behavioral plasticity.

Our results are consistent with previous suggestions that NA is necessary for cortical plasticity during development (e.g., Kasamatsu and Pettigrew, 1979; Sutherland et al., 1982; Castro et al., 1986; Loeb et al., 1987) and possibly in adulthood (e.g., Feeney and Sutton, 1987). Furthermore, our results confirm studies showing that depletion of cortical NA reduces brain size and alters dendritic morphology in normal animals (e.g., Felton
AREA TE APICAL DENDRITES

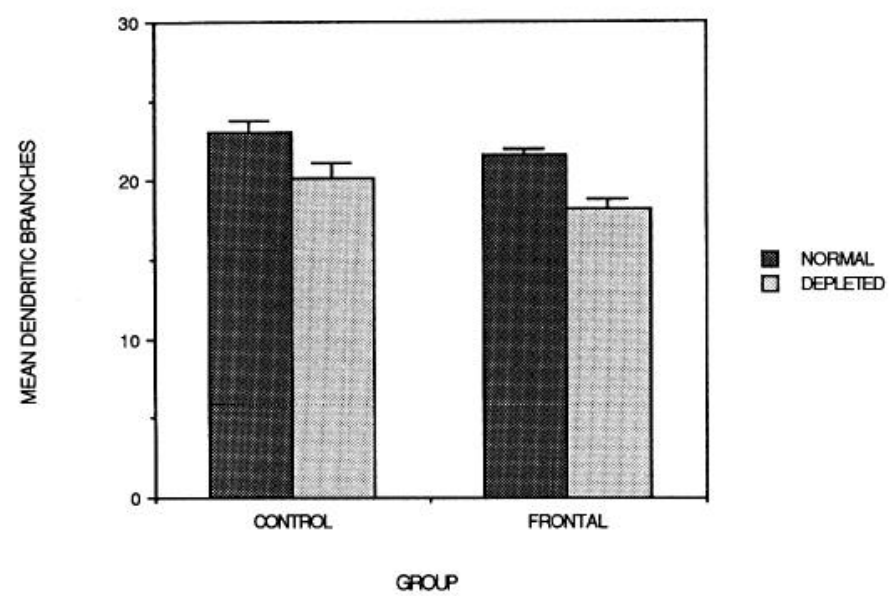

AREA TE BASILAR DENDRITES

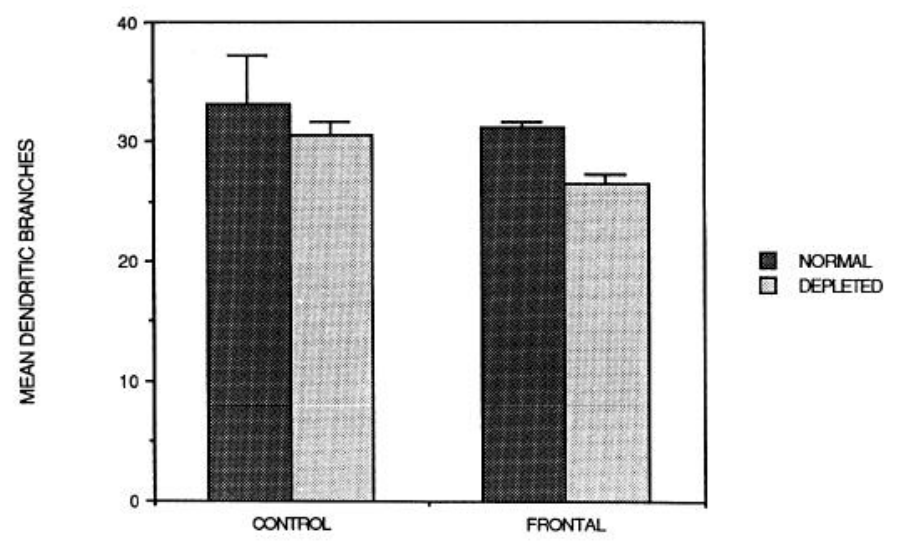

GROP

Figure 7. Mean number of apical and basilar dendritic branches for pyramidal cells in layer II/III of area TE2. The 6HDA treatment (DEPLETED) reduced branching in both control and neonatal frontal lesion groups, but the effect was greater in the frontal lesion group.

et al., 1982; Loeb et al., 1987). In addition, our results show that NA depletion interacts with neonatal frontal lesions to produce an even smaller brain, thinner cortex, and restricted dendritic branching than does either treatment alone. We must note, however, that in contrast to the Felton et al. (1982) study, which showed marked morphological abnormalities in Golgistained cortical neurons of neonatally 6HDA-treated animals, we did not observe such structural malformations. Rather, the cortical neurons of the 6HDA-treated animals were normal in general appearance, although they were visually (and quantifiably) simpler with respect to the extent of dendritic branching. The principal methodological difference between the Felton et al. study and the present one was rat strain, as the former authors used albino rats and we used hooded rats. Similarly, Lidov and Molliver (1982) used hooded rats in their study of the effects of prenatal 6HDA lesions, and they also found no dendritic abnormalities.

The differential effect of frontal lesions at P4 and P7 is consistent with earlier studies showing that there is no sparing of function after bilateral cortical lesions in rats between P1 and P5, but there is significant sparing after lesions at P7-P12 (Kolb 
A Sutherland et al (1982)

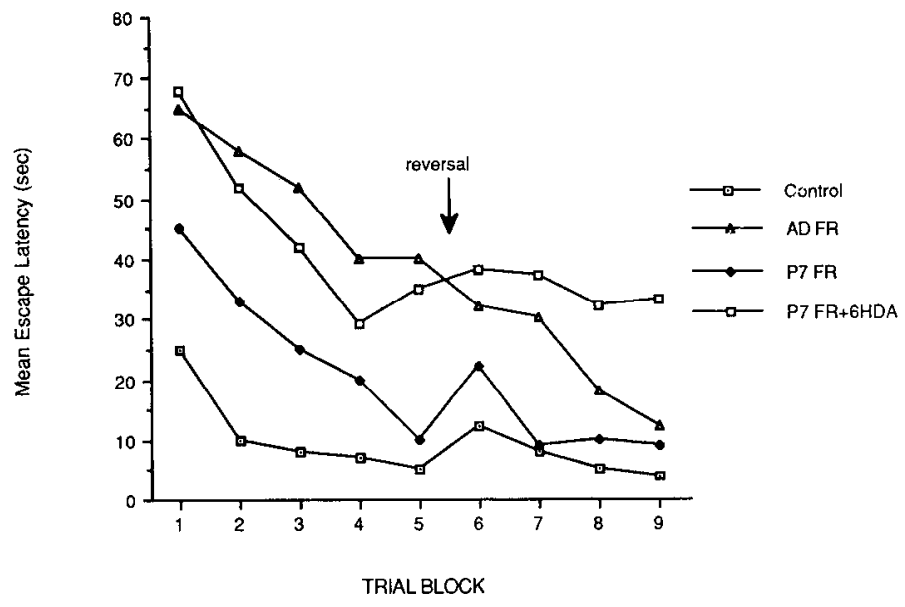

B P7 Replication

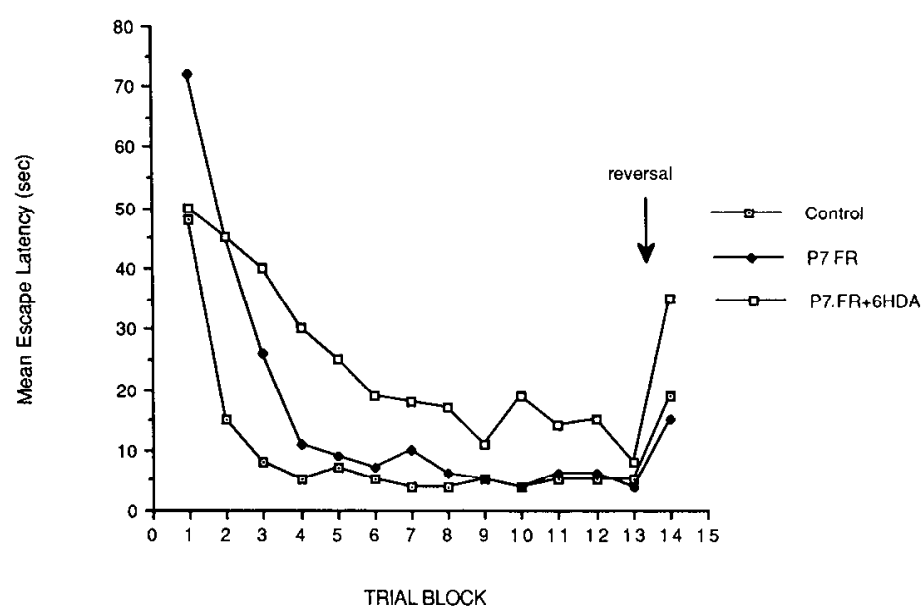

Figure 8. A, Mean latency on blocks of four trials to find the hidden platform in the Morris water task in experiment 1. For clarity, the scores of the two adult-operated groups have been pooled as they did not differ significantly. The arrow indicates when the platform was moved. Rats with lesions at $\mathrm{P} 7(P 7 F R)$ performed better than adult frontal operates $(A D F R)$ and NA-depleted neonatal frontal operates $(P 7 F R+6 H D A)$. $B$, Mean latency to find the hidden platform in experiment 2 . The animals in this experiment were tested with an additional five trial blocks, and the platform was not moved until trial block 14. As in experiment 1 , the NA-depleted neonatal frontal lesion rats performed significantly worse than the saline-treated frontal lesion rats.

and Whishaw, 1981b, 1985; Kolb, 1987). This suggests that there may be some type of "critical period" for cortical plasticity around P7-P12, and it is at this time that NA is especially important to plasticity (Fig. 10). The basis of the critical period is unknown, although it could be related to the fact that there are few cortical astrocytes before about $\mathrm{P} 7$, with the principal period of astrocytogenesis being from about P7-P15 (NietoSampedro and Cotman, 1985). Astrocytes have been shown to play a role in recovery from cortical injury, and their activity in adulthood is alleged to be influenced by NA (Coleman and Flood, 1988). Thus, it is possible that the mechanisms underlying functional sparing are dependent upon astrocyte activity, which are in turn influenced by noradrenergic activity. In the absence of NA, the astrocyte response to injury in the infant brain may be altered, reduced, or absent.
A Escape Latency

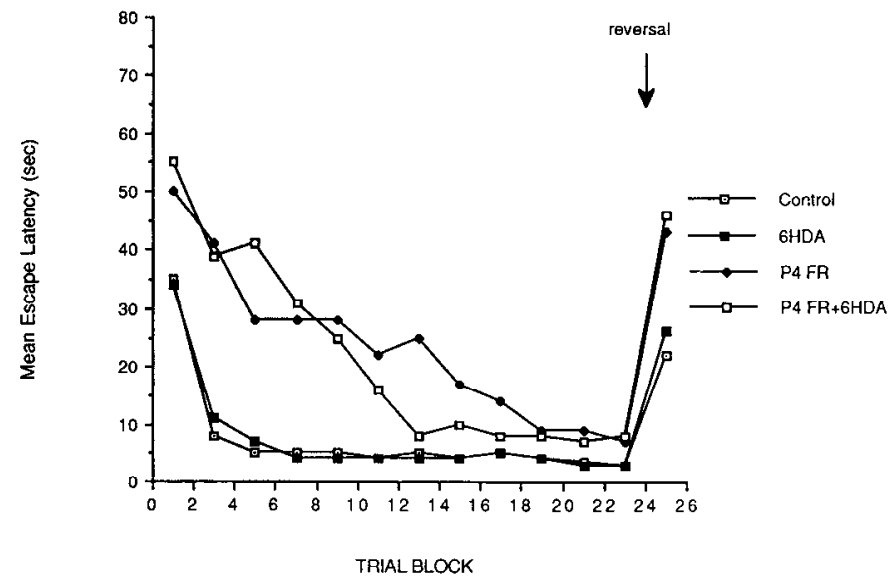

B Heading Errors

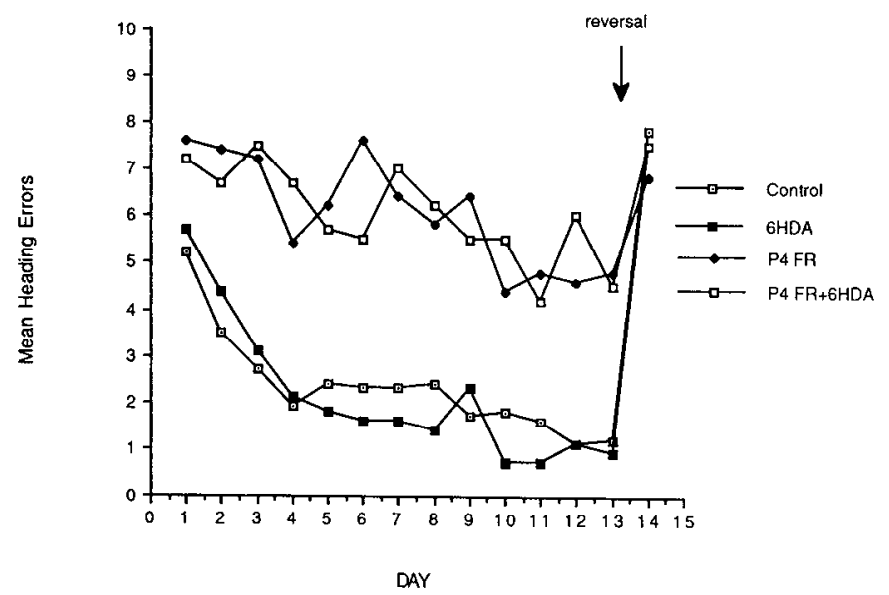

Figure 9. A, Mean latency to find the hidden platform in the Morris water task in experiment 3.6HDA treatment had no significant effect upon the performance of either unoperated control rats or rats with frontal lesions on P4. The P4 frontal lesion rats eventually learn to find the platform fairly quickly, but the error analysis $(B)$ shows that they do not swim directly to the platform and are unlikely to have learned its location. Abbreviations are as in Figure 4.

Kolb and Gibb (1991a,b) proposed that increased dendritic branching might reflect a general mechanism of cortical plasticity, although the precise nature of this mechanism was not specified. Hence, they noted that increased dendritic branching was correlated with recovery from cortical injury in adulthood, sparing from cortical injury in infancy, and improved maze performance after enriched rearing. The present results showed that rats with frontal lesions at $\mathrm{P} 7$ showed partial sparing of spatial maze learning without greater dendritic branching than normal animals, which would appear to be inconsistent with their hypothesis. Four points are relevant here. First, frontal cortical injury before P7 is associated with a decrease in dendritic branching and a complete absence of sparing (Kolb and Gibb, 1991a). Second, frontal lesions at $\mathbf{P 7}$ are associated with task-dependent sparing and an increase in dendritic branching relative to animals with earlier lesions. Depletion of NA completely blocks this sparing and produces a markedly reduced dendritic arbor. Third, frontal lesions at P10 are correlated with a massive increase in dendritic branching and extensive behavioral sparing. Fourth, enriched rearing allows sparing after P5 


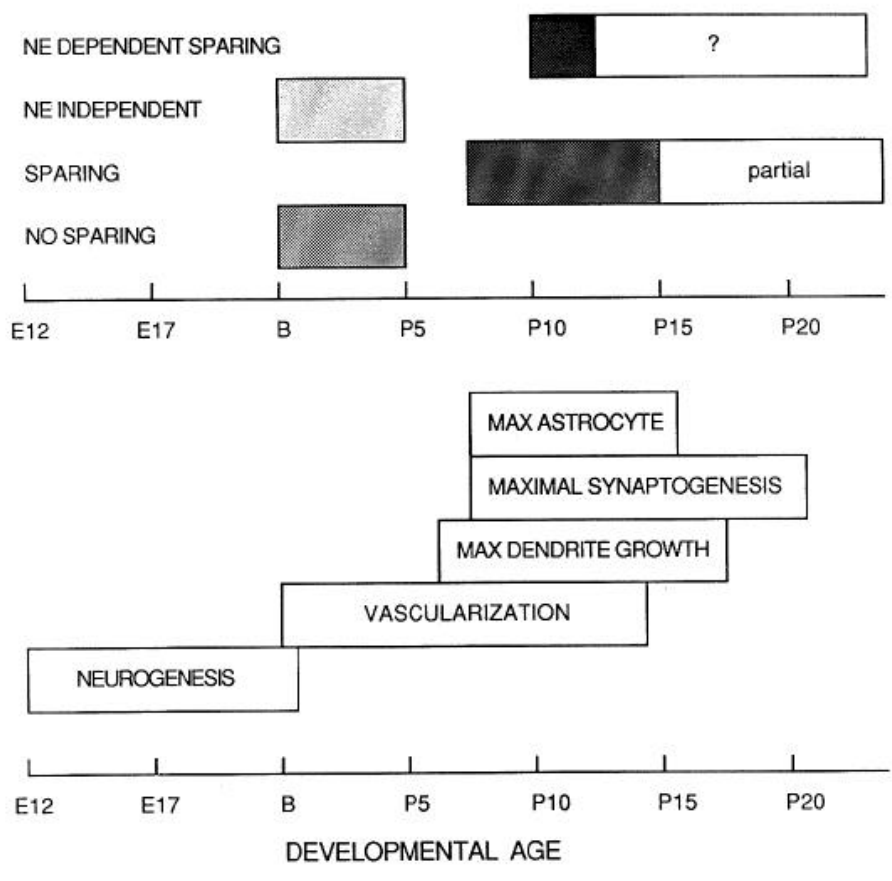

Figure 10. Time course of events during development of the neocortex. The boxes in the bottom portion indicate the period of maximum development of neurons (neurogenesis), blood vessels (vascularization), dendritic growth, synapse formation, and astrocyte formation (after Nieto-Sampedro and Cotman, 1985). The boxes in the top portion indicate the developmental periods during which there is or is not behavioral sparing after cortical lesions, as well as the periods during which NA depletion blocks or does not effect sparing. The abscissa indicates developmental age, beginning at embryonic day 12 and continuing until P25.

lesions (Kolb and Elliott, 1987) and is correlated with a larger increase in cortical thickness and dendritic branching than is observed in control animals reared in enriched environments (Kolb and Elliott, 1987). Taken together, these results can be seen as supportive of the general notion that changes in dendritic arborization reflect some mechanism underlying cortical plasticity. Nonetheless, the correlation of partial sparing and no increase in dendritic branching relative to controls in the rats with frontal lesions on $\mathrm{P} 7$ implies that there must be additional mechanisms supporting the observed behavioral sparing. These mechanisms remain to be identified.

\section{References}

Brenner E, Mirmiran M, Uylings HBM, van der Gugten J (1983) Impaired growth of the cerebral cortex of rats treated neonatally with 6-hydroxydopamine under different environmental conditions. Neurosci Lett 42:13-17.

Castro AJ, Kartje-Tillotson G, Barnes D, Swenson RS (1986) Disruption of corticospinal plasticity by 6-hydroxydopamine as measured by intracortical microstimulation in rats. Dev Brain Res 24: 295-298.

Coleman P, Flood DG (1988) Is dendritic proliferation of surviving neurons a compensatory response to loss of neighbors in the aging brain? In Brain injury and recovery (Finger S, LeVere TE, Almli CR, Stein DG, eds), pp 245-248. New York: Plenum.

Coleman PD, Riesen AH (1968) Environmental effects on cortical dendritic fields: I. Rearing in the dark. J Anat 102:363-374.

Feeney DM, Sutton RL (1987) Pharmacotherapy for recovery of function after brain injury. CRC Crit Rev Neurobiol 3:135-197.

Felice LJ, Felice JD, Kissinger PT (1978) Determination of catecholamines in rat brain parts by reverse phase ion-paired liquid chromatography. J Neurochem 31:1461-1465.
Felton DL, Hallman H, Jonsson G (1982) Evidence for a neurotrophic role of noradrenaline neurons in the postnatal development of rat cerebral cortex. J Neurocytol 11:119-135.

Gordon B, Allen EE, Trombley PQ (1988) The role of norepinephrine in plasticity of visual cortex. Prog Neurobiol 30:171-191.

Kasamatsu T, Pettigrew JD (1976) Depletion of brain catecholamines: failure of ocular dominance shift after monocular occlusion in kittens. Science 194:206-209.

Kasamatsu T, Pettigrew JD (1979) Preservation of binocularity after monocular deprivation in the striate cortex of kittens treated with 6-hydroxydopamine. J Comp Neurol 185:139-162.

Kasamatsu T, Pettigrew JD, Ary M (1979) Restoration of visual cortical plasticity by local microperfusion of norepinephrine. J Comp Neurol 185:163-181.

Kolb B (1987) Recovery from early cortical damage in rats. I. Differential behavioral and anatomical effects of frontal lesions at different ages of neural maturation. Behav Brain Res 25:205-220.

Kolb B, Elliott W (1987) Recovery from early cortical lesions in rats: II. Effects of experience on anatomy and behavior following frontal lesions at 1 or 5 days of age. Behav Brain Res 26:47-56.

Kolb B, Gibb R (1991a) Sparing of function after neonatal frontal lesions correlates with increased cortical dendritic branching: a possible mechanism for the Kennard effect. Behav Brain Res 43:51-56.

Kolb B, Gibb R (1991b) Environmental enrichment and cortical injury: behavioral and anatomical consequences of frontal cortex lesions. Cerebr Cortex 1:189-198.

Kolb B, Whishaw IQ (1981a) Decortication in rats in infancy or adulthood produced comparable functional losses on learned and speciestypical behaviors. J Comp Physiol Psychol 95:468-483.

Kolb B, Whishaw IQ (1981b) Neonatal frontal lesions in the rat: sparing of learned but not species-typical behavior in the presence of reduced brain weight and cortical thickness. J Comp Physiol Psychol 95:863-879.

Kolb B, Whishaw IQ (1985) Earlier is not always better: behavioral dysfunction and abnormal cerebral morphogenesis following neonatal cortical lesions in the rat. Behav Brain Res 17:25-43.

Kolb B, Sutherland RJ, Whishaw IQ (1983a) Abnormalities in cortical and subcortical morphology after neonatal neocortical lesions in rats. Exp Neurol 79:223-244.

Kolb B, Sutherland RJ, Whishaw IQ (1983b) A comparison of the contributions of the frontal and parietal association cortex to spatial localization in rats. Behav Neurosci 97:13-27.

Kolb B, Day J, Gibb R, Whishaw IQ (1989) Recovery from early cortical lesions in rats: 6 . Cortical noradrenaline, cortical thickness, and development of spatial learning after frontal lesions or hemidecortication. Psychobiology 17:370-376.

Lidov HGW, Molliver ME (1982) The structure of cerebral cortex in the rat following prenatal administration of 6-hydroxydopamine. Dev Brain Res 3:81-108.

Loeb EP, Chang F-LF, Greenough WT (1987) Effects of neonatal 6-hydroxydopamine treatment upon morphological organization of the posteromedial barrel subfield in mouse somatosensory cortex. Brain Res 403:113-120.

Mohammed AK, Jonsson G, Archer T (1986) Selective lesioning of forebrain noradrenaline neurons at birth abolishes the improved maze learning performance induced by rearing in complex environment. Brain Res 398:6-10.

Morris RGM (1981) Spatial localization does not require the presence of local cues. Learn Motivation 12:239-260.

Murtha S, Pappas BA, Raman S (1990) Neonatal and adult forebrain norepinephrine depletion and the behavioral and cortical thickening effects of enriched/impoverished environment. Behav Brain Res 39: 249-261.

Nieto-Sampedro M, Cotman CW (1985) Growth factor induction and temporal order in central nervous system repair. In: Synaptic plasticity (Cotman CW, ed), pp 407-455. New York: Guilford.

O'Shea L, Saari M, Pappas BA, Ings R, Stange K (1983) Neonatal 6-hydroxydopamine attenuates the neural and behavioral effects of enriched rearing in the rat. Eur J Pharmacol 92:43-47.

Pappas BA, Saari M, Peters D, Roberts DCS, Fibiger HC (1978) Neonatal systemic 6-hydroxydopamine and dorsal tegmental bundle lesion: comparison of effects on CNS norepinephrine and the postdecapitation reflex. Brain Res 155:205-208.

Robinson TE, Becker JB, Young EA, Akil H, Castenada E (1987) The effects of footshock stress on regional brain dopamine metabolism 
and pituitary B-endorphin release in rats previously sensitized to amphetamine. Neuropharmacology 26:679-691.

Shirokawa T, Kasamatsu T (1986) Concentration-dependent suppression by B-adrenergic antagonists of the shift in ocular dominance following monocular deprivation in kitten visual cortex. Neuroscience 18:1035-1046.

Shirokawa T, Kasamatsu T, Kuppermann BD, Ramachandran VS (1989) Noradrenergic control of ocular dominance plasticity in the visual cortex of dark-reared cats. Dev Brain Res 47:303-308.

Sutherland RJ Kolb B, Whishaw IQ, Becker JB (1982) Cortical noradrenaline depletion eliminates sparing of spatial learning after neonatal frontal cortex damage in the rat. Neurosci Lett 32:125-130.

Sutherland RJ, Whishaw IQ, Kolb B (1983) A behavioural analysis of spatial localization following electrolytic, kainate- or colchicineinduced damage to the hippocampal formation in the rat. Behav Brain Res 7:133-153.

Uylings HBM, Kuypers K, Diamond MC, Veltman WAM (1978) Effects of differential rearing on plasticity of dendrites of cortical pyramidal neurons in adult rats. Exp Neurol 62:658-677.

Whishaw IQ, Sutherland RJ, Kolb B, Becker J (1986) Effects of neonatal forebrain noradrenaline depletion on recovery from brain damagc: pcrformance on a spatial navigation task as a function of age of surgery and postsurgical housing. Behav Neural Biol 46:285-307.

Zilles K (1985) The cortex of the rat: a stereotaxic atlas. New York: Springer. 